\title{
Gas detection by quartz enhanced photoacoustic spectroscopy
}

\author{
Michal Dostal ${ }^{1,2}$, Jan Suchanek ${ }^{1,2}$, Vaclav Valek ${ }^{2}$, Vaclav Nevrly², Petr Bitala ${ }^{2}$, Simona Slivkova ${ }^{2}$, \\ Eliska Kristlova ${ }^{2}$, Pavel Kubat ${ }^{1}$, Pavel Janda ${ }^{1}$, Svatopluk Civis $^{1}$, Zdenek Zelinger ${ }^{1}$ \\ ${ }^{1} \mathrm{~J}$. Heyrovsky Institute of Physical Chemistry of the CAS, v. v. i., Prague, Czech Republic,

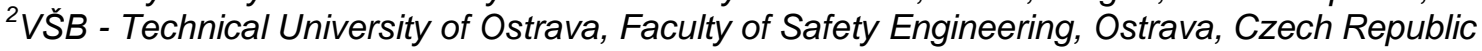 \\ michal.dostal@jh-inst.cas.cz
}

\begin{abstract}
Photoacoustic spectroscopy is a detection method in which the light source energy is converted to sound [1], subsequently the sound captured by microphone. This method can be used for quantitative chemical analysis at ultra-low concentrations of gaseous species or samples with low absorption coefficient. Quartz enhanced photoacoustic spectroscopy replaces the classical conventional microphone and enables rapid and highly sensitive detection of trace gas concentrations, when using quartz tuning fork with a high quality factor [2]. Tunable quantum cascade laser partly covering acetonitrile infrared spectra [3] was employed in this study as an excitation source for quartz enhanced photoacoustic spectroscopy. Vapor of acetonitrile was used as measuring gas. From the practical point of view this gas is of importance as a marker of explosive compounds such as trinitrotoluene and hexogen [4], or it can be released into the atmosphere during combustion of biomass [5]. Case specific design of the photoacoustic cell and experimental setup for the purposes of quantum cascade laser - quartz enhanced photoacoustic spectroscopy detection is finally reported within this work.
\end{abstract}

Key words: spectroscopy, photoacoustic spectroscopy, gas sensing, QEPAS cell

\section{Introduction}

Air quality measurement and detection of dangerous substances constitutes an integral part of improving the environment and raising the level of safety. It puts the emphasis on the development of sensitive and selective gas detectors.

Photoacoustic spectroscopy (PAS) is based on the detection of photoacoustic phenomenon which was first observed by Alexander Graham Bell in the year 1881 [1]. Acoustic pressure resulting from the conversion of absorbed light energy to the kinetic energy of translational motion of molecules in the gas can be easily measured using a microphone [6].

This work describes the development and testing gas detector using Quartz enhanced photoacoustic spectroscopy (QEPAS).

\section{Experimental}

The sensitivity PAS is limited pressure sensitivity element (usually microphone) employed for detecting acoustic waves. Enhanced sensitivity can be achieved by replacing the classical microphone micromechanical element with a high $Q$ factor (quality factor) [7].
In this experiment quartz tuning fork (QTF) is assumed the sensitive acoustic wave detector. Shifted resonate frequency of QTF can signalize the acoustic wave propagation. Commercial QTF set included the controller unit (NanoAndMore Tuning Sensor Controller) used in the experiment which is purpose at atomic force microscope (AFM).

Tunable Quantum cascade laser (QCL) was utilized as a source of infrared (heat) radiation. Laser was modulated by an external arbitrary function generator (Siglent SDG 1025) with the sine function. Laser beam was focused into the central part of QEPAS cell using optmechanical elements.

The cell made of stainless steel that was used for this experiment is suitable for two variants of QEPAS. First one method: QTF oscillator was situated between two micro-resonators, second one: one micro-resonator and QTF placed out of the laser beam [8]. Modification of cell from one method to the other is possible because of $90^{\circ}$ cylinder turning. The 3D model of cell can be seen as shown in Figure (1). 


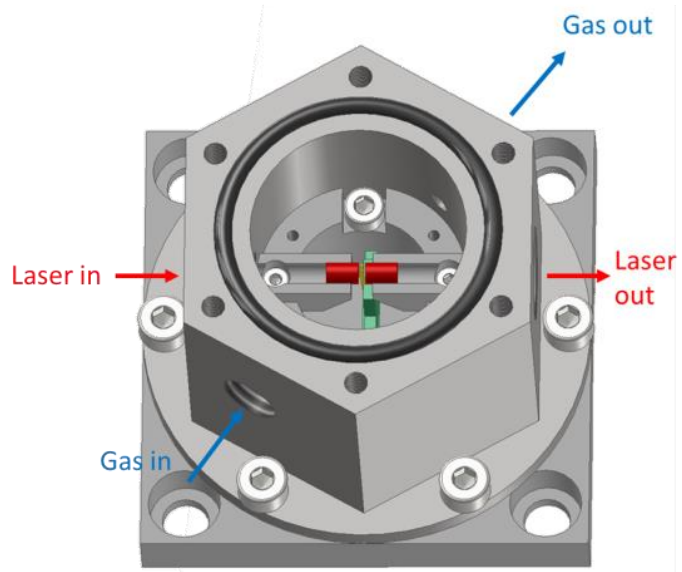

Fig. 1: 3D model of QEPAS cell.

Cell allows measurement in the flow and flooding gas into the cell and its closure. Microresonators are tubes made of stainless steel, which help to increase the sensitivity of the system. In case of two micro-resonators QTF is placed between them and the laser bean passes through the tips of the forks. The method with one micro-resonators in the middle of it, created a small hole and fork is placed next to the hole. Laser beam passes through the micro-resonator. Method with one microresonator was used for the first test experiments described in this article. Microresonator was made from stainless steel tube (Swagelok) with a diameter of $D=1 / 16$ " length $L=8 \mathrm{~mm}$ with an aperture of diameter. This size was chosen as the best [8].

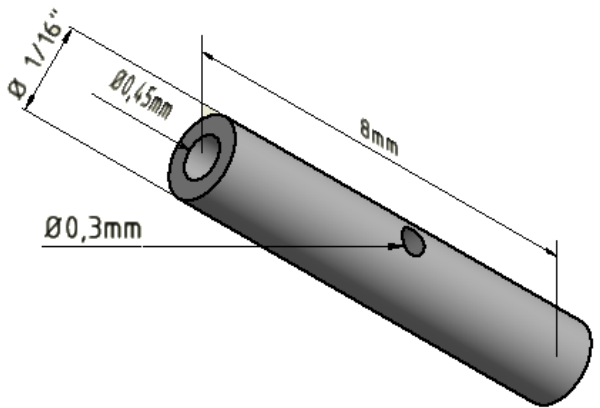

Fig. 2: Micro-resonator tube for off beam method.

Quartz tuning fork is a control unit. It can vibrate main resonant frequency and simultaneously it can measure output signal. This signal is displayed using by oscilloscope (LeCroy 9361) and demodulated phase-sensitive lock-in amplifier (Stanford Research Systems SR530 lock-in amplifier). The demodulated $2 f$ signal from lock-in amplifier is recorded and processed in the PC by analog/digital converter. The basic experimental arrangement is shown in Figure (3). List of the equipments for creation and processing photoacoustic signal is displayed in this arrangement.

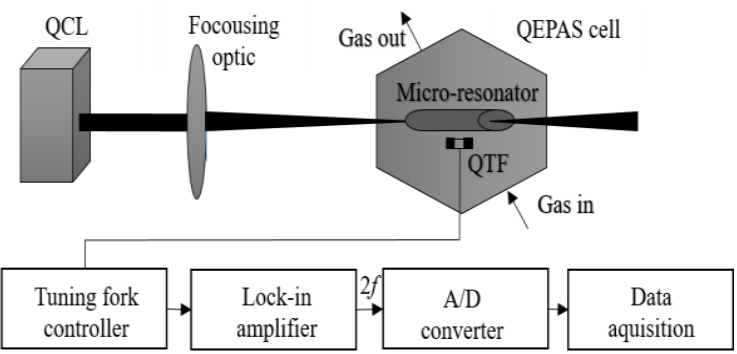

Fig. 3: Base experimental setup.

\section{Result and discussion}

At beginning experiments leaded to build and set up and control the system's response. The controlling resonant frequency QTF was the first experiment. And also resonant frequency leaded to correctly settings preamplifier QTF.

Generator of function was connected to the control unit and it enabled function "sweep". Medium frequency $f$ was set to $f=32.755 \mathrm{kHz}$, frequency range $f=2 \mathrm{kHz}$ and the sweep time $t=5 \mathrm{~s}$. Narrow peak resonance frequency QTF was measured by oscilloscope as shown in Figure (4).

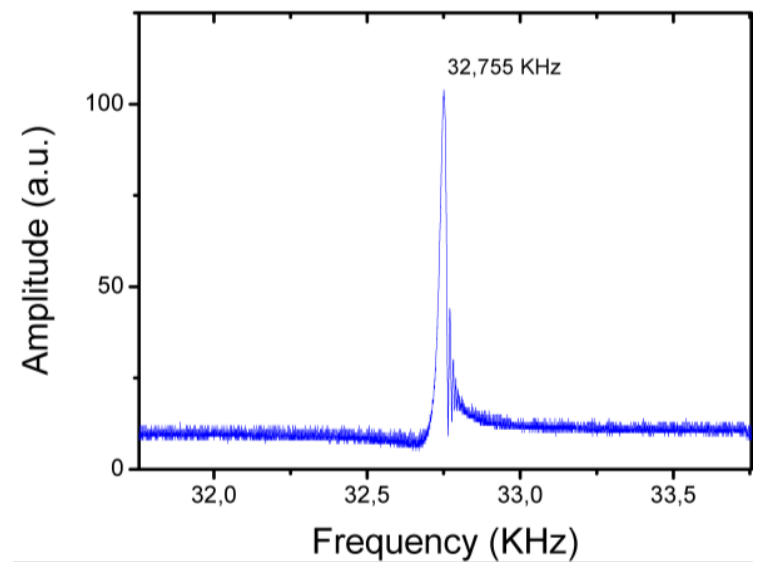

Fig. 4. Frequency Scan.

Another experiment was QTF response to generate vibration from an external source. For this experiment we used piezoceramic (PZT) oscillator which was placed on cell in Figure (5). PZT oscillator should input voltage modulated at a frequency of $f_{o s}=37.35 \mathrm{kHz}$. Originate mechanical vibration. 


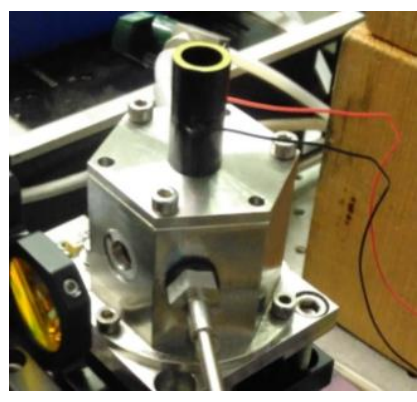

Fig. 5: QEPAS cell with PZT oscillator.

The result was the occurrence of delta waves during measurement signal from QTF as shown in Figure (6).

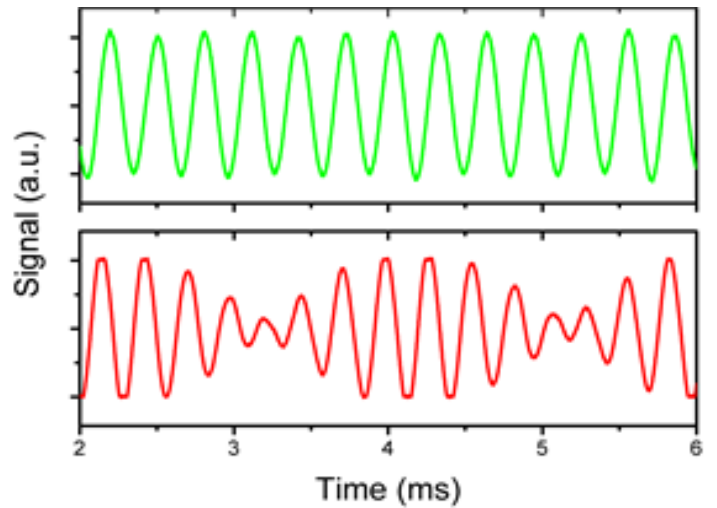

Fig. 6: The measured output signals from the QTF, without influenced PZT (green), influenced by the PZT oscillator (red).

These measurements show response QTF to the vibratory wave from an external source. In the same way, there should be a change in absorption of IR radiation in the gas. As a test gas was used acetonitrile having several spectral lines assigned to R-branch of the fundamental $U_{4}$ band where was measured by laser diode spectroscopy (will be posted in another article). In this measurement was used QCL laser with wavenumber between 935.4 to $936.8 \mathrm{~cm}^{-1}$, corresponding to the abovementioned strip of the spectral lines. The measurements also directed to calibrate a laser so that a certain value of the current source corresponds to a particular wavelength of radiation. Calibration was done by comparison the measured spectrum with the spectrum of gas simulated using spectraplot web-application (www.spectraplot.com).

\section{Conclusion}

First QEPAS spectrometer in Prague is in the development stage and this work describes a preparatory measure and show structural elements of the system. In this paper we show the first measurements described QTF response to an external source of vibration. The next phase will be followed by measurement of gas detection and setting detection limits of the system. From the results will be evaluated by suitable construction to the design QEPAS cell to increase the sensitivity of the system. Ultimately, we want to build autonomous sensor which will be controlled by microcomputer. Finally, these sensors will be compiled to sensor network for air quality monitoring.

\section{Acknowledgements}

The authors are grateful to the financial support from Project No. 14-14696S funded by the Czech Science Foundation and Project No. LD14022 within COST Action TD1105 funded by the Ministry of Education, Youth and Sports of the Czech Republic.

\section{References}

[1] A. Bell, The Production of Sound by Radiant Energy, Science, 2(48), 242-253 (1881); http://www.jstor.org/stable/2900190.

[2] A. A. Kosterev, F. K. Tittel, D. V. Serebryakov, A. L. Malinovsky, I. V. Morozov, (2005), Applications of quartz tuning forks in spectroscopic gas sensing, Review of Scientific Instruments, 76(4), (2005); http://dx.doi.org/10.1063/1.1884196.

[3] A. M. Tolonen, M. Koivusaari, R. Paso, J. Schroderus, S. Alanko, R. Anttila, The Infrared Spectrum of Methyl Cyanide Between 850 and $1150 \mathrm{~cm}-1$ : Analysis of the $v_{4}, v_{7}$, and $3 v^{1} 8$ Bands with Resonances, Journal of Molecular Spectroscopy, 160(2), 554-565 (1993); doi:10.1006/jmsp.1993.1201.

[4] T. Osborn, S Kaimal, W. Burns, A. R. Ford, S. W. Reeve, Spectral signatures for volatile impurities in TNT and RDX based explosives, InSPIE Defense and Security Symposium (2008); doi:10.1117/12.777844.

[5] R. Holzinger, J. Williams, G. Salisbury, T. Klüpfel, M. de Reus, M. Traub, P. J. Crutzen, J. Lelieveld, Oxygenated compounds in aged biomass burning plumes over the Eastern Mediterranean: evidence for strong secondary production of methanol and acetone, Atmospheric Chemistry and Physics 5, 39-46 (2005); doi:10.5194/acp-539-2005.

[6] Z. Zelinger, M. Střižík, P. Kubát, S. Civiš, Quantitative analysis of trace mixtures of toluene and xylenes by $\mathrm{CO}_{2}$ laser photoacoustic spectrometry, Analytica chimica acta, 422(2), 179-185 (2000); http://dx.doi.org/10.1016/S00032670(00)01069-2.

[7] J. Kauppinen, K. Wilcken, I Kauppinen, V. Koskinen, High sensitivity in gas analysis with photoacoustic detection, Microchemical journal, 76(1), 151-159 (2004); http://dx.doi.org/10.1016/j.microc.2003.11.007.

[8] L. Kun, G. Xiaoyong, Y. Hongming, Ch. Weidong, Z. Weijun, G. Xiaoming, Off-beam quartzenhanced photoacoustic spectroscopy, Optics Letters, 34, 1594-1596 (2009); https://doi.org/10.1364/OL.34.001594. 